\title{
An unusual case of Aspergillus endocarditis of native aortic valve following ECMO
}

\author{
S Nanditha ${ }^{1} \cdot$ GaneshKrishnan K T Iyer $^{2} \cdot$ B Raghu $^{1} \cdot$ Y M Prashanth ${ }^{2} \cdot$ Vikas Yadav $^{1}$ \\ Received: 5 December 2019 / Revised: 12 March 2020 / Accepted: 20 March 2020 / Published online: 11 May 2020 \\ (C) Indian Association of Cardiovascular-Thoracic Surgeons 2020
}

\begin{abstract}
Fungal endocarditis following Extracorporeal membrane oxygenation (ECMO) is rare and very rarely reported. Though rare it has a high mortality rate. A 49-year-old male patient who had venovenous ECMO presented with aortic valve endocarditis after 40 days. He underwent sutureless bioprosthehtic valve placement and debridement of abscess. Abscess fluid grew aspergillus species and was started on dual antifungal intravenous amphotericin and variconazole. He was discharged after 1 month with oral variconazole. Incidence of aseptic endocarditis (AE) has been on rise due to increase in invasive procedures. Blood culture is mostly sterile, and fever may be absent. Abscess debridement and aggressive dual antifungal treatment helped our patient in his road to recovery. In current era with increasing use of ECMO, newer rarer complications should be kept in mind. High index of suspicion is required for diagnosing fungal endocarditis after ECMO.
\end{abstract}

Keywords Aspergillus endocarditis $\cdot$ Native valve $\cdot$ ECMO complication

\section{Introduction}

Fungal endocarditis is rare in clinical practice. Fungal endocarditis forms a minor portion of $1-10 \%$ among all cases of infective endocarditis (IE) [1]. Though rare, it has an alarmingly high mortality rate of about 50\% [2]. Extracorporeal membrane oxygenation (ECMO) usage in intensive care unit for pulmonary and cardiac support has been on a rise. Infections in the form of bacterial pneumonia (33\%) and sepsis $(29 \%)$ are major complications after ECMO [3]. Fungal endocarditis following ECMO is rare and we have not found any reported case in our literature search.

GaneshKrishnan K T Iyer

gktiyer@gmail.com

S Nanditha

nanditha.iyengar88@gmail.com

B Raghu

rag_anaesthesia@rediffmail.com

Vikas Yadav

drvikaskumayadav@yahoo.co.in

1 Department of Cardiac Anesthesia, Aster CMI, Bangalore, India

2 Department of Cardiothoracic Surgery, Aster CMI, Bangalore, India

\section{Case report}

A 49-year-old male patient with morbid obesity was admitted in our center with sudden onset breathlessness. Screening echocardiography showed right atrial and right ventricular dilatation with estimated pulmonary artery (PA) systolic pressure of $70 \mathrm{mmHg}$. Computed tomography pulmonary angiography diagnosed massive pulmonary embolism. He was thrombolyzed with injection reteplase. On venous Doppler, deep vein thrombosis was diagnosed. In view of worsening respiratory distress, he was put on mechanical ventilation and veno-venous ECMO. The right internal jugular vein and right femoral vein were cannulated by an ultrasound-guided percutaneous technique. He was decannulated after 6 days. During his 2-month stay in the hospital, he underwent hemodialysis for acute renal failure, had multiple antibiotics for sepsis and steroids in the form of dexamethasone $8 \mathrm{mg}$ thrice daily for 5 days, and had tracheostomy. Twenty days after ECMO was terminated, he was weaned off ventilator and tracheostomy. Patient conditions improved and he was discharged with sterile blood cultures. Before discharged, his echo showed normal PA pressures and normal aortic valve morphology. He was ambulatory while discharged and needed support while walking. On discharge, he was advised to continue tablet dabigatran $150 \mathrm{mg}$ twice daily for long term. 
Forty-three days after discharged, the patient presented with numbness in the right hand, slurring of speech, and blurring of vision in the left eye. Doppler evaluation showed no flow in the radial artery and slow flow in the ulnar artery. Magnetic resonance imaging (MRI) of the brain showed acute lacunar infarcts in bilateral fronto-parietal lobes. The patient did not have any fever spikes and blood culture came sterile. The left eye was diagnosed to have endophthalmitis and the patient received topical antibiotics and topical steroids. Screening transthoracic echo done on the 3rd day showed vegetation on the aortic valve with paravalvular abscess. He had mild eccentric aortic regurgitation with normal PA pressures and normal left ventricular systolic function. He was started electively on broad-spectrum antibiotics. However, the patient next day developed complete heart block and increase in abscess size on echo. He was taken up for emergency surgery and diseased aortic valve was replaced with sutureless bioprosthetic valve. Operative findings showed tricuspid aortic valve with vegetations at the right and non-coronary cusp junction and abscess at the aortic annulus extending into the interventricular septum. Extensive debridement of abscess was done and sent for culture. The raw area was covered with pericardial patch. The patient was weaned off cardiopulmonary bypass with dopamine, adrenaline, and noradrenaline support. He was extubated after $72 \mathrm{~h}$.

The patient continued to receive intravenous vancomycin and meropenem. Culture of abscess fluid showed growth of Aspergillus fumigatus. Based on sensitivity, he was started on intravenous liposomal amphotericin $500 \mathrm{mg}$ once daily which he received for 15 days and intravenous voriconazole $600 \mathrm{mg}$ twice daily. In the interim period, the patient was maintaining own heart rate of about $65-70 / \mathrm{min}$ in complete heart block. He was put on backup mode with epicardial pacing leads. Serial blood cultures were sent and after ensuring negative culture, the patient underwent permanent pacemaker implantation (PPI) on 12th postoperative day. After PPI, echo showed normally functioning aortic valve prosthesis with paravalvular leak. Grade 1 + mitral regurgitation was noted with fair left ventricular function and ejection fraction of $50 \%$. Gradient across the prosthetic valve was $18 / 11 \mathrm{mmHg}$ and PA pressure was $20 \mathrm{mmHg}$. After 1 month of hospital stay, the patient was discharged with sterile blood cultures and normally functioning prosthetic valve. $\mathrm{He}$ was told to continue oral voriconazole for 6 months and to review then. He was started on tablet warfarin to maintain International normalized ratio (INR) of about $2-2.5$ and plan to switch to oral dabigatran after 3 months. The patient continues to come for regular follow-ups and is doing well.

\section{Discussion}

Nosocomial IE is defined when it's contracted $>48 \mathrm{~h}$ and within 3 months after admission into acute care facility [4].
It is typically caused by nosocomial pathogens like enteric gram-negative bacilli and Staphylococcus aureus but nosocomial fungal IE has been on the rise [1]. Fungal endocarditis (FE) is most commonly caused by Candida and Aspergillus species. Candida endocarditis has incidence of $24-46 \%$ among fungal species and Aspergillus forms $25 \%$ of all cases of FE [5].

Risk factors for fungal endocarditis include parenteral nutrition, immunosuppression, underlying cardiac abnormalities, prosthetic heart valves, indwelling central venous catheters, prolonged use of broad-spectrum antibiotics, and cardiovascular surgery [6]. Most common complications after ECMO are renal failure (56\%), bacterial pneumonia (33\%), and sepsis (26\%) [3]. After arterio-venous ECMO, most common complications have been related to bleeding $(41 \%)$ and infection (49.5\%) [6]. Fungal endocarditis has not been documented after ECMO.

Incidence of aseptic endocarditis has been on the rise due to increase in invasive procedures. It is extremely difficult to diagnose and high index of suspicion is required. Blood culture is mostly sterile and fever may be absent [4]. In our case as well, the patient did not have any fever spikes and blood culture was sterile. The initial symptom was upper limb numbness due to embolism which is significantly associated with AE [4].

In view of increasing paravalvular abscess size and complete heart block, the patient was taken up for surgery. On the fourth postoperative day, when abscess culture showed Aspergillus species, dual antifungal was initiated. Amphotericin was given for 15 days and voriconazole was continued after discharge as well. During his stay, blood culture developed Klebsiella, and sensitive antibiotics were administered. Before shifting, he was blood culture sterile.

The patient also had endophthalmitis and in spite of vigorous antibiotics and intravitreal injections, vision in the left eye was lost.

\section{Conclusion}

In the current era with increasing use of ECMO, newer, rarer complications should be kept in mind. High index of suspicion is required for diagnosing fungal endocarditis after ECMO.

\section{Compliance with ethical standards}

Ethical approval Not applicable.

Funding None.

Conflicts of interest Authors do not have any conflict of interest. 
Informed consent Informed consent was obtained.

Statement of Human and animal rights Not applicable.

\section{References}

1. Giamarellou H. Nosocomial cardiac infections. J Hosp Infect. 2002;50:91-105.

2. Pierrotti LC, Baddour LM. Fungal endocarditis, 1995-2000. Chest. 2002;122:302-10.

3. Zangrillo A, Landoni G, Biondi-Zoccai G, et al. A meta-analysis of complications and mortality of extracorporeal membrane oxygenation. Crit Care Resusc J Australas Acad Crit Care Med. 2013;15:172-8.

4. Meshaal MS, Labib D, Said K, et al. Aspergillus endocarditis: diagnostic criteria and predictors of outcome, a retrospective cohort study. PLoS One. 2018;13:e0201459.

5. Yuan S-M. Fungal endocarditis. Braz J Cardiovasc Surg. 2016;31: 252-5.

6. Koerner MM, Harper MD, Gordon CK, et al. Adult cardiac venoarterial extracorporeal life support (VA-ECMO): prevention and management of acute complications. Ann Cardiothorac Surg. 2019;8:66-75.

Publisher's note Springer Nature remains neutral with regard to jurisdictional claims in published maps and institutional affiliations. 